\title{
Material multimedia para aumentar la motivación y el aprendizaje en el aula universitaria
}

Eva Serna $^{a}$, Javier Megías ${ }^{b}$, Gloria Olaso ${ }^{a}$, María Dolores Mauricio ${ }^{\text {a }}$ y Teresa SanMiguel $^{\mathrm{b}}$

a Departamento de Fisiología, Universitat de Valencia. Eva.serna@uv.es, ํDepartamento de Patología, Universitat de Valencia. Teresa.Miguel@uv.es

\begin{abstract}
The multimedia material used as a teaching medium in the classroom can help the teaching-learning process of the students. It also helps the teacher in his work of transmitting information from another point of view more striking and entertaining. This would cause an enlargement and enrichment in the recipient's experience to assimilate more quickly, dynamically and fluidly. In this study we analyze the grades obtained with and without the help of a multimedia material prepared by teachers. Subsequently, the opinion of the students is collected through an anonymous and voluntary survey allowing the teachers to collect the information necessary for the search to improve the quality of teaching.
\end{abstract}

Keywords: multimedia material, evaluation, quality of university education.

\footnotetext{
Resumen

El material multimedia usado como medio didáctico en el aula puede ayudar al proceso enseñanza-aprendizaje del alumnado. Además le sirve de apoyo al profesor en su labor de trasmitir la información desde otro punto de vista más llamativo y entretenido. Esto provocaría una ampliación y enriquecimiento en la experiencia del receptor para asimilar el contenido didáctico de manera más rápida, dinámica y fluida. En este estudio se analizan las calificaciones obtenidas con y sin ayuda de un material multimedia elaborado por profesores. Posteriormente, se recoge la opinión del alumnado mediante una encuesta anónima y voluntaria permitiendo al profesorado recoger la información necesaria para a partir de ésta, elaborar unos objetivos de mejora de la calidad de la enseñanza.
}

Palabras clave: material multimedia, evaluación, calidad enseñanza universitaria. 


\section{Introducción}

La enseñanza universitaria necesita de nuevos recursos o medios para transmitir y presentar la información. Por ello el uso de material multimedia podría ser una de las estrategias didácticas que ayudarían en este sentido (Marquès, 2000). Al término material multimedia lo entendemos como el medio que utiliza conjunta y simultánemanete diversos medios, como imágenes, sonidos y texto en la transmisión de la información.

La motivación es un factor determinante del rendimiento académico (Roncel, 2007). Entendemos la motivación como un "incentivo, necesidad o deseo que el alumno siente de aprender y alcanzar un nivel elevado en la meta", concepto en que intervienen cuatro componentes: fijarse un objetivo, el deseo de alcanzarlo, actitudes favorables hacia una actividad particular y esfuerzo. Se trata de un concepto, en el que las variables psicosociales, la motivación y la actitud hacia el aprendizaje son mucho más decisivas que otros factores como la inteligencia, ya que las primeras son más susceptibles de modificación (Luján-García, 1999). Además, para la mayoría de investigadores, es la imagen animada, un entorno gráfico atractivo para el educando, el elemento motivador por excelencia, habiéndose demostrado que favorece la retención (Salinas, 1996).

Además de proporcionar información, el material multimedia puede orientar al estudiante, remarcando los conceptos más importantes, reforzando los puntos más conflictivos o aumentando la motivación, uno de los principales motores del aprendizaje (Polanco, 2005).

A la hora de incorporar estos recursos a la educación superior, es necesario o bien formar a los docentes para que sepan crear su propio material multimedia o bien ofrecer un servicio de calidad que se encargue de la parte técnica y oriente al profesor en la elaboración del mismo. En la Universitat de València existen convocatorias anuales de proyectos de innovación educativa, que en una de sus modalidades ofrecen la ayuda técnica para elaborar un material multimedia. Nuestro equipo docente desarrolló un proyecto a cargo de la mencionada convocatoria durante el curso académico 2015-2016 (Projectes innovació Código proyecto: UV-SFPIE_RMD15-377257). En nuestro caso el material elaborado entraría dentro de la definición de presentación multimedia.

Este tipo de recurso multimedia apoyaría al profesor en su labor de estimular el deseo de aprender y aumentar la autoestima del alumno y es, por tanto, una buena opción para ayudar en el proceso enseñanza-aprendizaje obteniendo mejores resultados. Por ello, "entender mediante conceptos y entender a través de la vista se combinan en una suma positiva reforzándose o al menos integrándose el uno en el otro" (Sartori, 1998), lo que viene a reforzar la tesis de que la cultura escrita y la cultura audiovisual dan lugar a una síntesis armoniosa.

(cc) EY-NC-ND 2018, Universitat Politècnica de València

Congreso IN-RED (2018) 


\section{Objetivos}

El objetivo general es la valoración de un material multimedia de elaboración propia sobre el aprendizajey la motivación del alumnado.

Objetivos específicos:

1. Comparar las calificaciones obtenidas por los equipos del curso 2016-2017 que realizaron la actividad sin la ayuda del material multimedia, con las obtenidas por los equipos del curso 2017-2018 que han tenido a su disposición el material multimedia.

2. Analizar la encuesta anónima y voluntaria sobre el material multimedia proporcionado.

3. Recopilar la opinión del alumnado sobre el impacto del material multimedia.

\section{Desarrollo de la innovación}

La experiencia se llevó a cabo con el alumnado matriculado en la asignatura de Biología Celular y Tisular de $1^{\text {er }}$ Curso del Grado de Podología de la Universitat de València en el curso académico 2017-2018 (Código de Asignatura: 34324 con 6 ECTS y con carácter de formación básica). El número total de alumnos fue 55 de 67 matriculados.

Mediante el uso del microscopio óptico podemos describir los componentes que integran la célula y que son descritos en la parte teórica de la asignatura. Así, podemos ayudar al alumnado a consolidar sus conocimientos teóricos y permitir familiarizarse con su uso para distinguir y caracterizar las estructuras celulares.

La elaboración del material multimedia se dividió en tres partes:

1. Descripción y manejo óptimo de las partes del microscopio óptico.

Se detalla el nombre y función de cada parte, tanto mecánica como óptica, y se explica su manejo adecuado dentro de un laboratorio.

MMEDIA: http://mmedia.uv.es/buildhtml/45978

RODERIC: http://roderic.uv.es/handle/10550/62959

YOUTUBE: https://youtube/86RmR6Lpo1c

2. Obtención de un frotis sanguíneo y tinción de Giemsa.

Esta etapa es fundamental para que el alumnado que nunca ha trabajado en un laboratorio pueda ver cómo se procede a la obtención de una preparación biológica. Además la tinción de Giemsa es el método habitual para el examen de frotis sanguíneos, cortes histológicos, y otros tipos de muestras biológicas. Por tanto, se explica una técnica básica para que en el futuro sea puesta en práctica en su carrera profesional.

MMEDIA: http://mmedia.uv.es/buildhtml/46889

(c) ) EY-NC-ND 2018, Universitat Politècnica de València

Congreso In-Red (2018) 
RODERIC: http://roderic.uv.es/handle/10550/56145

YOUTUBE: https://youtube/rmKx5JWmo4I

3. Observación de tipos celulares.

En esta parte, procedemos a observar al microscopio óptico, mediante la ayuda de un proyector, la preparación realizada en la etapa 2 . En esta fase les enseña a diferenciar los tres tipos celulares que van a encontrar en la sangre: eritrocitos, leucocitos y plaquetas.

MMEDIA: http://mmedia.uv.es/buildhtml/46662

RODERIC: http://roderic.uv.es/handle/10550/62960

YOUTUBE: https://youtube/gQwqS5xEEhE

Este material multimedia fue realizado gracias a la concesión de un proyecto de Innovación Docente titulado "Elaboración de material docente multimedia para la realización de prácticas de laboratorio enfocadas al uso de microscopio óptico y observación de tipos celulares” con Código: UV-SFPIE_RMD15-377257 y coordinado por Eva Serna García.

La encuesta que se les proporcionó, tras la visualización del material multimedia, para realizarla de forma anónima y voluntaria fue la que se muestra en la Figura 1. 


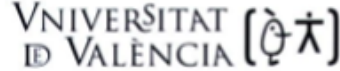 \\ Facultat de Medicina i Odontologia \\ Departament de Patologia}

\section{Encuesta sobre el material multimedia proporcionado por la Unidad de Biología}

1-¿'Te ha gustado?

\begin{tabular}{|l|l|l|l|l|l|}
\hline Nada & Poco & Indiferente & Bastante & Mucho & NS/NC \\
\hline & & & & & \\
\hline
\end{tabular}

2-¿Resulta ameno?

\begin{tabular}{|l|l|l|l|l|l|}
\hline Nada & Poco & Indiferente & Bastante & Mucho & NS/NC \\
\hline & & & & & \\
\hline
\end{tabular}

3-¿Resulta largo?

\begin{tabular}{|l|l|l|l|l|l|}
\hline Nada & Poco & Indiferente & Bastante & Mucho & NS/NC \\
\hline & & & & & \\
\hline
\end{tabular}

4-¿Aprecias tener este material de apoyo?

\begin{tabular}{|l|l|l|l|l|l|}
\hline Nada & Poco & Indiferente & Bastante & Mucho & NS/NC \\
\hline & & & & & \\
\hline
\end{tabular}

5-¿Te ha servido para reforzar la parte práctica y entender mejor la parte teórica de la asignatura

\begin{tabular}{|l|l|l|l|l|l|}
\hline Nada & Poco & Indiferente & Bastante & Mucho & NS/NC \\
\hline & & & & & \\
\hline
\end{tabular}

6-Propón alguna sugerencia de mejora o algún tema que te gustaría tener mediante esta metodología multimedia. (Respuesta libre).

Fig. 1 Encuesta anónima para la valoración de los alumnos sobre el material multimedia distribuido

\section{Resultados}

Los resultados obtenidos sobre las calificaciones del alumnado se recogen en la Tabla 1. Como se puede apreciar, se obtuvo un incremento de más de $5 \%$ en las calificaciones de su examen referidas al contenido de prácticas, y por tanto, al contenido que el material multimedia complementaba en la formación del alumnado. 
Tabla 1. Porcentaje de respuestas acertadas en las preparaciones de sangre humana y de anguila de las dos poblaciones de estudio.

\begin{tabular}{cccc}
\hline Población & $\begin{array}{c}\text { \% Respuestas correctas } \\
\text { en preparaciones de } \\
\text { sangre humana }\end{array}$ & $\begin{array}{c}\text { \% Respuestas } \\
\text { correctas en } \\
\text { preparaciones de } \\
\text { sangre de anguila }\end{array}$ & $\begin{array}{c}\text { Cantidad total de } \\
\text { respuestas } \\
\text { computadas y \% } \\
\text { acertado }\end{array}$ \\
\hline $\begin{array}{c}\text { Sin MM (Curso 2016- } \\
\text { 2017) }\end{array}$ & $80,0 \%$ & $83,8 \%$ & 67 (82,1\% acertado) \\
Con MM (Curso 2017- & $88,9 \%$ & $86,1 \%$ & 72 (87,5\% acertado) \\
2018) & & & \\
\hline
\end{tabular}

Sin MM: estudiantes que no tuvieron el material multimedia.

Con MM: estudiantes que tuvieron el material multimedia.

Tras pasar la encuesta al alumnado, el 65,45\% (36 alumnos/as) valoró la experiencia como que le había gustado bastante la visualización de los 3 videos (Figura 2), al 54,55\% (30 alumnos/as) les resultó bastante amena (Figura 3), el 65,45\% (36 alumnos/as) apreció mucho disponer de este tipo de material multimedia (Figura 4). Además al 32,73\% (18 alumnos/as) les parecía un formato adecuado ya que no les pareció que fuera largo (Figura 5), y el 47,27\% (26 alumnos/as) valoraba que el material mutimedia ayudaba a reforzar el contenido teórico-práctico dado en la asignatura (Figura 6).

\section{Te ha gustado}

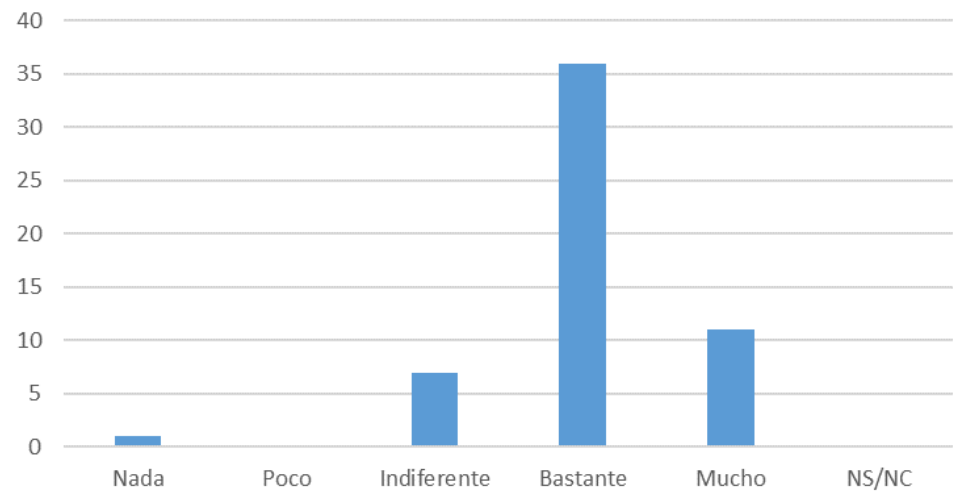

Fig. 2. Valoración del material multimedia a la pregunta de si "les ha gustado" de los 55 participantes. 


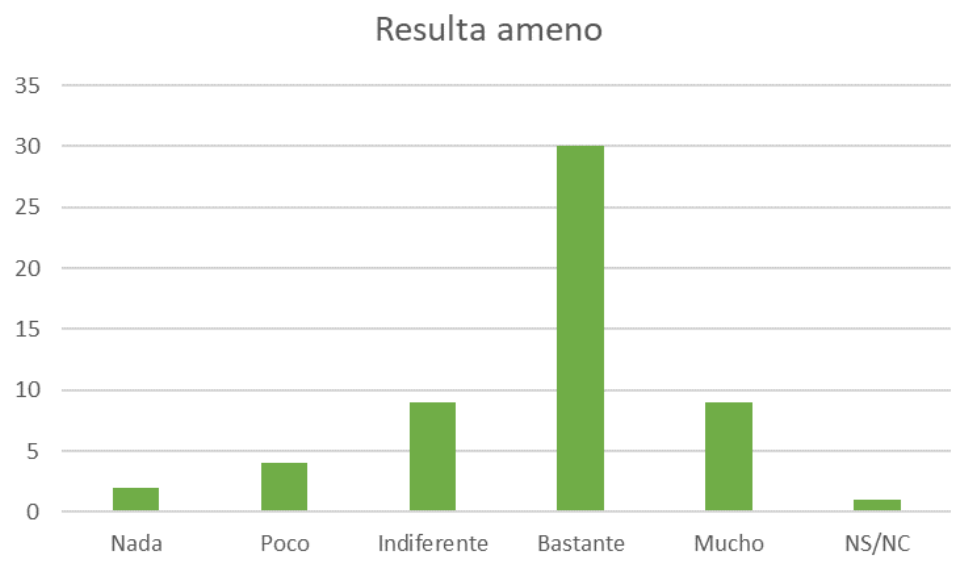

Fig. 3. Valoración del material multimedia a la pregunta de si "les ha resultado ameno" de los 55 participantes.

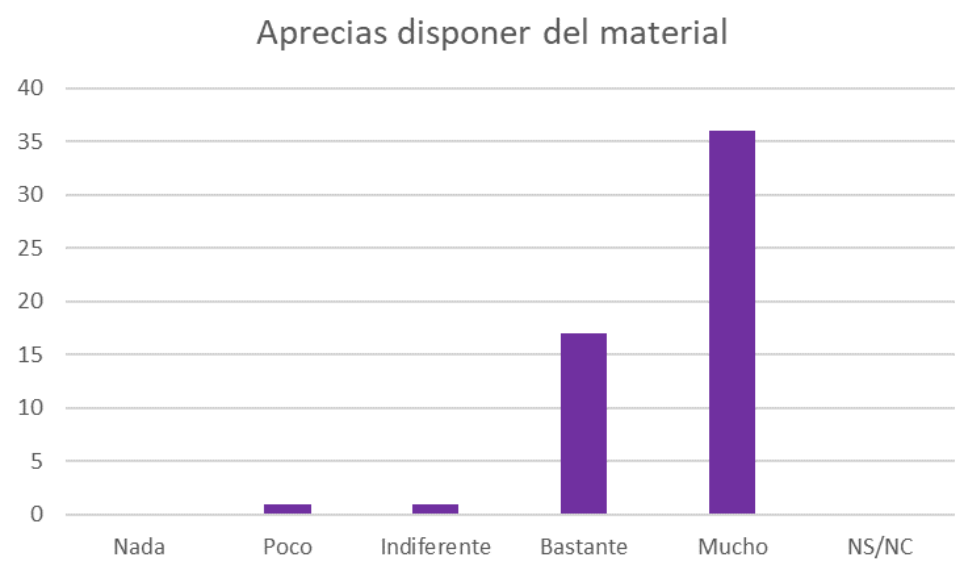

Fig. 4. Valoración del material multimedia a la pregunta de si "aprecias disponer del material” de los 55 participantes. 
Resulta largo

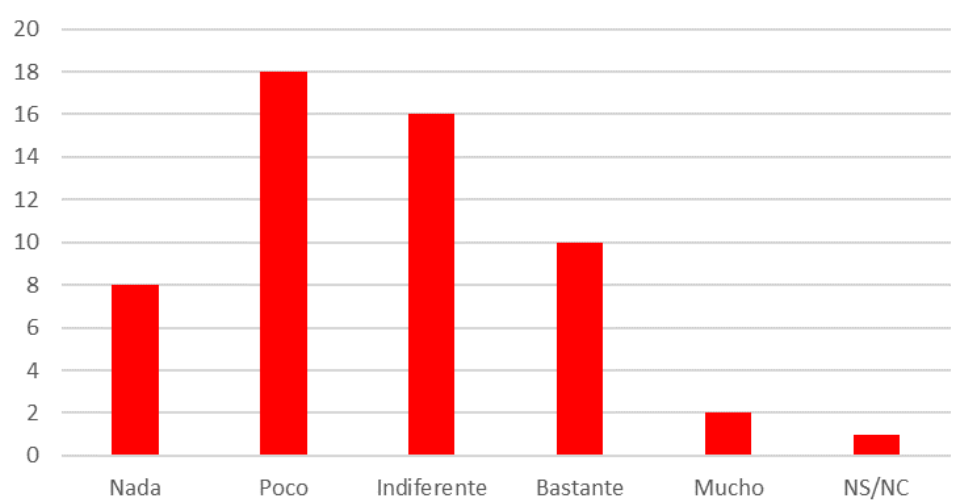

Fig. 5. Valoración del material multimedia a la pregunta de si "resulta largo" de los 55 participantes.

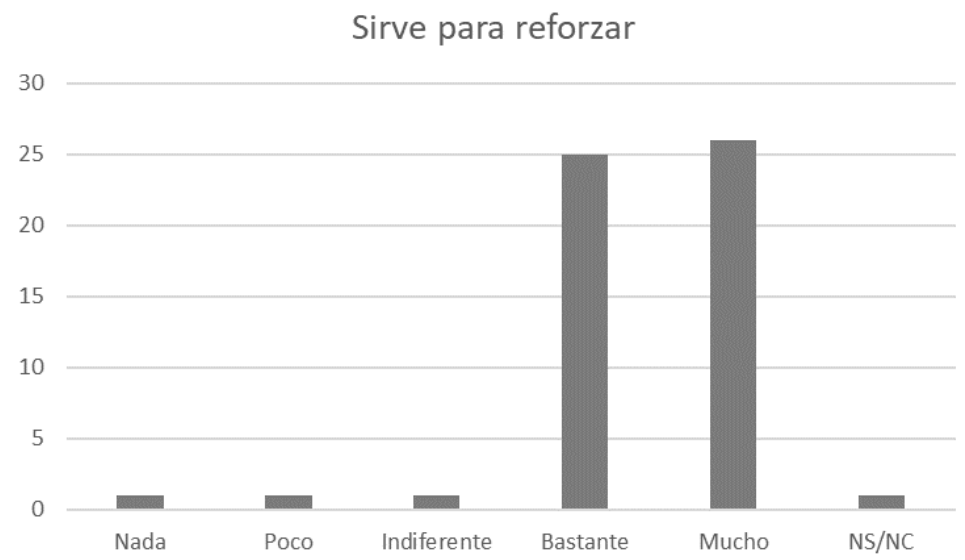

Fig. 6. Valoración del material multimedia a la pregunta de si "sirve para reforzar" de los 55 participantes.

Sobre la opinión libre de los alumnos (punto 6 de la encuesta proporcionada) obtuvimos un total de 19 aportaciones. De las cuales, destacamos que 8 opiniones que eran para pedir más material similar al elaborado y que también se realizara en el área, no solo de microscopia óptica, sino también de la electrónica. Tres opiniones estaban encaminadas a la interactividad a posteriori de la visualización del material, 2 pedían un cambio de música, y otros dos participantes opinaban que la duración fuera menor.

Además, contabilizamos la cantidad de visualizaciones de los tres vídeos realizados por las tres plataformas utilizadas para su divulgación (MMEDIA, RODERIC y YOUTUBE). La plataforma youtube fue la más utilizada, siendo el material mutimedia 2: “ Obtención de un frotis sanguíneo y tinción de Giemsa” el más exitoso, con más de 3.000 visitas.

(c)) EY-NC-ND 2018, Universitat Politècnica de València

Congreso IN-RED (2018) 


\section{Número de visualizaciones en la plataforma de youtube}

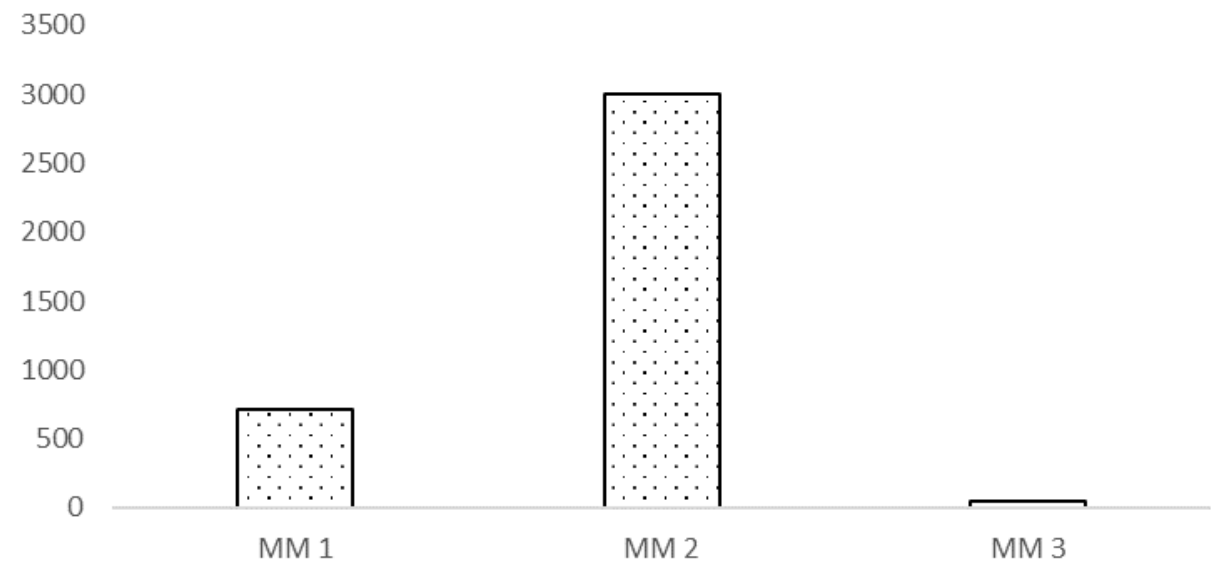

Fig. 7. Número de visualizaciones en la plataforma de youtube del material multimedia (MM) de cada uno de los vídeos elaborados (última actualización 13-03-2018.)

\section{Conclusiones}

La finalidad de nuestra experiencia es comprobar si con el uso de material multimedia, además de proporcionar al alumnado un recurso más atractivo desde el punto de vista del profesorado, si es interesante y aprovechoso para el alumnado y además si le potencia en su proceso de aprendizaje.

Desde un punto de vista de análisis de calificaciones, se pudo observar que el uso del material multimedia ayuda a mejorar significativamente el rendimiento del alumnado, ya que el aumento fue de más de $5 \%$.

La opinión del material multimedia realizado por el equipo de profesores y profesoras fue muy bien valorado, siendo para la mayoría del alumnado un recurso que gusta, es ameno, con el formato adecuado, y que es apreciado tenerlo para reforzar sus contenidos. Cabe destacar que este material introduce un factor de tipo motivacional que necesariamente favorece el aprendizaje.

De los tres videos generados, el que más éxito tiene, tanto para el alumnado como para los seguidores de la plataforma youtube, es el material multimedia correspondiente a la realización de un frotis sanguíneo y tinción de Giemsa (Material Multimedia 2). Este contenido se suele dar desde un punto de vista teórico y con la generación de este recurso se adentra al alumnado en un laboratorio real y le permite visualizar cada paso de manera detallada y relajada.

Como conclusión final podemos afirmar que el material multimedia es una herramienta que se debería usar en la docencia universitaria ya que tiene muy buena acogida por el alumnado y mejora en el rendimiento de su aprendizaje.

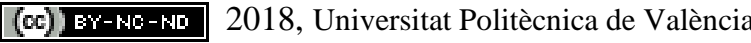




\section{Referencias}

LUJÁN-GARCÍA, C.I. (1999). La motivación: Un factor relevante en el proceso de enseñanza/aprendizaje de lenguas extranjeras. Boletín Millares Carlo, (18), 269-278.

MARQUÈS, P. (2000). Ventajas e inconvenientes del material multimedia educativo. Departamento de Pedagogía Aplicada, Facultad de Educación, UAB $<$ http://peremarques.net/ventajas.htm> [Consulta: 20 de marzo de 2018].

POLANCO, A. (2005). "La motivación en los estudiantes universitarios". Revista Electrónica "Actualidades Investigativas en Educación. Vol. 5, núm. 2, pp. 1-13 $<$ http://www.redalyc.org/articulo.oa?id=44750219 > [Consulta: 18 de marzo de 2018]

RONCEL VEGA, V. M. (2007). El rendimiento en ELE en la enseñanza reglada: un modelo explicativo. MarcoELE: Revista De Didáctica, (5).

SALINAS, J. (1996): "Multimedia en los procesos de enseñanza - aprendizaje: Elementos de discusión”. Ponencia en el Encuentro de Computación Educativa. Santiago de Chile, 2-4 mayo.

SARTORI, G. (1998) “Homo Videns, La sociedad teledirigida”. Ed. Aguilar, Altea, Taurus, Alfaguara, S.A., México. 